\title{
Evaluación de la interacción entre atención visual y memoria de trabajo: un estudio basado en técnicas de eye-tracking
}

\author{
Conesa, Natàlia ${ }^{a}$; Balagué, Marta ${ }^{a, b}$ y Dempere-Marco, Laura ${ }^{a^{*}}$ \\ ${ }^{a}$ Grupo de Investigación en Bioinformática y Estadística Médica, Departamento de Ingenierías \& \\ Departamento de Biociencias, Facultad de Ciencias y Tecnología, Universitat de Vic-Universitat \\ Central de Catalunya, \\ b Servicio de Neuropsicología, Hospital Moisès Broggi, Consorci Sanitari de Integral, (*) \\ laura.dempere@uvic.cat
}

\begin{abstract}
Working memory (WM) allows us to hold and manipulate information when it is no longer present to the physical senses. When stimuli are displayed sequentially, recency effects (i.e. stimuli presented later in the sequence are more likely held in WM) have been reported in visual WM. The main hypothesis that guides this study is that visual attention modulates the codification of visual information during the encoding phase of WM. Our results show that the recency effect emerges even when stimuli are shown simultaneously, thus affecting which stimuli enter and are maintained in WM. This effect emerges from the allocation of visual attention to the different items during the stimulation phase of the task, thus illustrating the important role of the interplay between visual attention and WM.
\end{abstract}

Keywords: working memory, attention, aging, eye-tracking, cognitive neuroscience.

\begin{abstract}
Resumen
La memoria de trabajo (MT) nos permite mantener y manipular información cuando ya no está presente a los sentidos. Cuando los estímulos se muestran de forma secuencial se ha descrito la aparición de efectos de recencia (i.e. aquellos estímulos que se muestran en las últimas posiciones de la secuencia son retenidos en MT con mayor probabilidad). La hipótesis principal que subyace este estudio es que la atención visual modula la codificación de información visual durante la fase de estimulación de MT. Nuestros resultados muestran que el efecto de recencia surge incluso cuando los estímulos se muestran de forma simultánea, de modo que determinan qué estímulos entran y son mantenidos en MT. Este efecto es consecuencia de la asignación de atención visual a los diferentes elementos durante la fase de estimulación de la tarea visual, ilustrando asi el importante rol de la interrelación entre atención visual y MT.
\end{abstract}


Palabras clave: memoria de trabajo, atención, envejecimiento, rastreo ocular, neurociencia cognitiva.

\section{Introducción}

La memoria de trabajo (MT) es la función cognitiva que nos permite mantener y manipular información cuando ya no está presente para los sentidos. Esta función cognitiva es básica para el desarrollo de otras funciones cognitivas superiores como la inteligencia, el lenguaje o la memoria (Cowan, 2017). Asimismo, la MT muestra un deterioro con la edad y con la presencia de diversas patologías relacionadas con el envejecimiento, entre ellas las neurodegenerativas. Uno de los mayores retos a los que se enfrenta nuestra sociedad se deriva del incremento en la esperanza de vida de las personas y la necesidad de acompañar la mayor longevidad de una buena calidad de vida. La función cognitiva es clave en este contexto para garantizar la calidad de vida y, por ello, es importante desarrollar técnicas que permitan la identificación de cambios sutiles en el rendimiento cognitivo de las personas. Esto es particularmente crítico en enfermedades como el Alzheimer o el Parkinson, con fases preclínicas muy largas, ya que es precisamente en estas fases en las cuales las terapias pueden ser más efectivas. Este trabajo constituye un avance en la caracterización de perfiles cognitivos, en particular de la MT, al introducir medidas de eyetracking que permiten analizar no sólo el rendimiento de las tareas sino también la dinámica de los procesos que conducen a tales rendimientos (Pallarés et al. 2016, 2017).

\section{Memoria de trabajo y atención visual}

Una característica distintiva de MT es su capacidad limitada. Diversos estudios sugieren que existe un límite absoluto de 3-4 elementos para estímulos visuales (Cowan, 2000, 2017). En la actualidad coexisten varias teorías sobre los mecanismos subyacentes que producen tales límites de capacidad. Los dos modelos principales son los modelos de capacidad fija (o modelos de slots) (Zhang y Luck, 2008) y los modelos de asignación dinámica (o modelos de recursos) (Bays y Husain, 2008). En los modelos de capacidad fija todos los elementos se recuerdan con la misma precisión hasta el límite (3-4 elementos), y se estima que no se almacenará información más allá de este límite. Por el contrario, en los modelos de asignación dinámica, los recursos (finitos) se reparten entre los elementos pero no necesariamente de la misma manera. Un aspecto destacable de este modelo es la predicción de que todos los elementos del conjunto de estimulación reciben ciertos recursos. En este contexto, la atención visual desempeña un rol crucial en la asignación de los recursos que recibe cada estímulo (Melcher y Piazza, 2011). En un estudio computacional Dempere-Marco et al. (Dempere-Marco et al., 2012) retomaron los resultados experimentales de Melcher y Piazza (Melcher y Piazza, 2011) e investigaron los 
mecanismos neuronales responsables de la capacidad limitada de MT y su relación con la relevancia visual, íntimamente relacionada con la atención visual. Tomando estos resultados como punto de partida, en este trabajo se amplía el paradigma experimental utilizado en estos estudios previos para investigar la relación entre la atención visual y la MT.

Para ello, se tiene en cuenta que cuando los estímulos se muestran secuencialmente emergen efectos seriales. En particular, en MT visual se ha observado el efecto de recencia (i.e. es más probable que se recuerden los estímulos presentados en las últimas posiciones de la secuencia). Por el contrario, el efecto de primacía (i.e. es más probable que se recuerden los estímulos presentados al principio de la secuencia) no se ha encontrado en MT visual, aunque sí se ha observado en MT verbal. En (Balagué y Dempere-Marco, 2016) se llevó a cabo un estudio computacional con el objetivo de investigar los mecanismos neuronales de la MT y la emergencia de los efectos seriales. Para estudiar la relación entre la atención visual y MT se ha diseñado un protocolo experimental de coincidencia con una muestra diferida (Delayed Match-to-Sample, DMS), monitorizado mediante técnicas de eye-tracking. Por un lado, se investiga cómo varía la MT con la edad y con el número de objetos presentes en la fase de estimulación. Posteriormente, se analiza hasta qué punto la atención visual modula la codificación de la información durante la fase de estimulación, lo que determina qué estímulos acceden y se mantienen en MT.

\section{Metodología}

\subsection{Descripción del experimento}

\subsubsection{Sujetos experimentales}

Un total de 54 sujetos participaron de forma voluntaria en los experimentos y fueron divididos en 2 cohortes: adultos jóvenes (J) (Edad: 24.10 土2.10 años; 13 hombres, 17 mujeres) y adultos de mediana edad (M) (Edad: 51.63 土5.03 años; 10 hombres, 14 mujeres). En ambas poblaciones, el número de sujetos con niveles de estudios similares fueron balanceados en ambas poblaciones ( $\mathrm{J}$ : 7 estudios medios, 23 estudios superiores; M: 8 estudios medios, 16 estudios superiores). Asimismo, se balanceó el ámbito de estudios (J: 13 artístico-social-humanístico, 17 científico-tecnológico; M: 9 artístico-socialhumanístico, 15 científico-tecnológico). Todos los sujetos participaron en una sesión experimental que se dividía en dos partes: 1) valoración neuropsicológica y 2) tarea DMS. 


\subsubsection{Valoración neuropsicológica}

Tal y como se ha indicado, los sujetos se sometieron a una valoración neuropsicológica exhaustiva con el objetivo de garantizar que su función coginitiva está completamente preservada, es decir, que se trata de individuos sanos desde la perspectiva cognitiva y sin ningún défícit/superávit cognitivo. Se llevaron a cabo un total de 10 pruebas neuropsicológicas en que se valoraron las siguientes funciones (por medio de los tests indicados entre paréntesis): vocabulario, atención y memoria de trabajo (span auditivo (Coalson et al. 2008) y Trial Making Test A (Tamayo, 2012, Peña-Casanova et al. 2009a), habilidades ejecutivas (fluencia semántica (Casals-Coll, 2013; Peña Casanova et al. 2009c ) fluencia verbal (Casals-Coll, 2013; Peña-Casanova et al., 2009c), Stroop (Golden, 1978; Rognoni, 2004; Peña-Casanova, 2009b) y Trial Making Test B (Tamayo, 2012; PeñaCasanova et al., 2009a), memoria (RAVLT (Spreen et al., 2006)), procesamiento de información (clave de números (Coalson et al., 2008)) y habilidades visuoconstructivas (cubos (Coalson et al., 2008)).

\subsubsection{Diseño experimental: tarea DMS monitorizada con eye-tracking.}

Todos los sujetos participaron en una tarea DMS (ver Fig. 1) que consiste en una serie de estímulos cuadrados de colores que se presentaron simultáneamente en matrices de 2, 4 o 6 elementos durante $1000 \mathrm{~ms}$. En este protocolo experimental todos los estímulos se encuentran a la misma distancia del centro de la pantalla (5.5 grados visuales) y, en cada ensayo, hay la misma cantidad de estímulos en cada hemiespacio. Tras $2 \mathrm{~s}$ sin estimulación, se presenta un estímulo de prueba en el centro de la pantalla, durante $0.5 \mathrm{~s}$, para su posterior reconocimiento. El sujeto dispone de $5 \mathrm{~s}$ para contestar por medio de dos botones: verde (objeto presente en conjunto de memorización), o rojo (objeto no presente en conjunto de memorización). Cada experimento consta de 252 ensayos presentados al azar en un diseño equilibrado en el número de estímulos, el color, la ubicación espacial y el estímulo de reconocimiento. Durante el experimento, la mirada de los sujetos fue monitorizada por medio de un eye-tracker (Tobii X120, $f=120 \mathrm{~Hz}$ ). Los estímulos se mostraron en una pantalla de tamaño $33.8 \mathrm{~cm} \times 27 \mathrm{~cm}$ con una resolución de $1280 \times 1080$ y que estaba a una distancia fija de $60 \mathrm{~cm}$ del eye-tracker. Se utilizó un soporte reposabarbillas con el objetivo de minimizar los movimientos de la cabeza durante el experimento, garantizando así un buen mantenimento de la calibración, al tiempo que ofrece una mayor comodidad a los participantes. Los 252 ensayos de dividieron en 3 bloques de 84 ensayos cada uno también balanceados en el número de estímulos, el color, la ubicación espacial y la presencia/ausencia del estímulo de reconocimiento en el conjunto de memorización. 


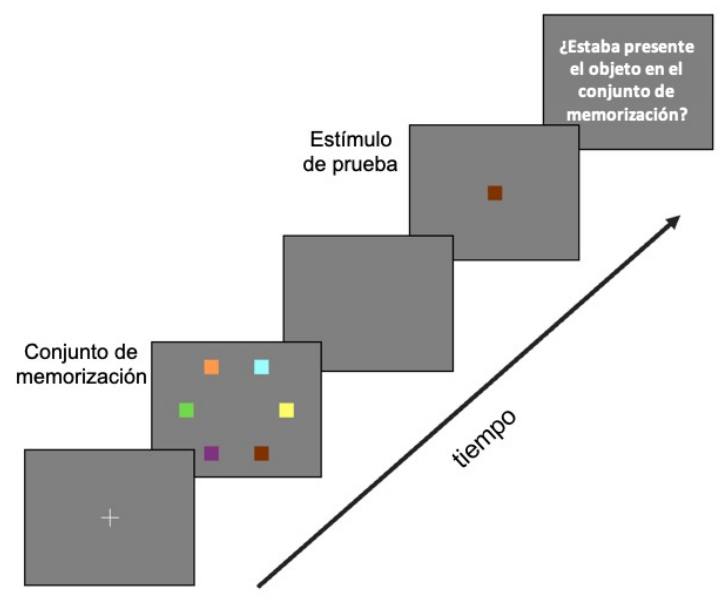

Fig. 1: Ilustración de la tarea DMS. Tras $500 \mathrm{~ms}$ de estabilización de la mirada en una cruz central, se muestra durante $1 \mathrm{~s}$ (fase de codificación/estimulación) el conjunto de memorización (matriz de 2, 4 o 6 de cuadrados de distintos colores ubidados a 5.5 grados visuales del centro). Posteriormente, durante 2 s (fase de memorización sin estímulos) se muestra una pantalla sin estímulos. Al acabar, se muestra el estímulo de prueba durante $500 \mathrm{~ms}$ (fase de prueba), seguido de la pregunta: “Estaba presentes el objeto en el conjunto de memorización?”. El suejo experimental dispone de 5 s para contestar. Todo el ensayo es monitorizado mediante un eye-tracker Tobii X120.

Los colores de los estímulos fueron definidos en un estudio previo de modo que fuesen claramente distinguibles entre ellos al tiempo que se minimizasen los efectos de prominencia visual (visual saliency) ya que pueden sesgar los resultados comportamentales. La descripción cuantitativa de los colores en sus componentes RGB se describen en la Tabla 1.

Tabla 1. Descripción cuantitativa de los colores de los estímulos en el espacio RGB.

\begin{tabular}{cccc}
\hline Color & R & G & B \\
\hline Naranja & 1 & 0.6 & 0.3 \\
\hline Verde & 0.45 & 0.85 & 0.3 \\
\hline Lila & 0.5 & 0.2 & 0.5 \\
\hline Amarillo & 1 & 1 & 0.4 \\
\hline Cian & 0.6 & 1 & 1 \\
\hline Marrón & 0.5 & 0.2 & 0 \\
\hline Rosa & 1 & 0.6 & 1 \\
\hline
\end{tabular}




\subsection{Análisis de datos}

\subsubsection{Rendimiento en la tarea}

De la tarea DMS, independientemente de los datos de eye-tracking, se han evaluado una serie de parámetros con el objetivo de investigar si existe algún sesgo comportamental en función del color de los estímulos visuales (errores en función del color), si varía el rendimiento en función del número de estímulos (errores en función del número de estímulos), la tipología de respuestas correctas e incorrectas (matriz de confusión), si hay aprendizaje a lo largo del experimento (evolución de la proporción de errores a lo largo del experimento) así como la dinámica particular de cada tipo de respuesta (tiempo medio de respuesta en función del tipo de respuesta). En particular, se han llevado a cabo los siguientes estudios:

- Rendimiento global. Análisis de la frecuencia de aciertos y errores de ambas poblaciones experimentales.

- Errores en función del número de estímulos. Errores que cometen los sujetos experimentales según el número de estímulos que aparecen en la fase de memorización.

- Matriz de confusión. Se distinguen cuatro posible respuestas (ver Tabla 2): positivos verdaderos (PV; el estímulo test está presente en el conjunto de memorización y el sujeto experimental responde correctamente que está presente), falsos positivos (FP; el estímulo test no está presente en el conjunto de memorización y el sujeto experimental responde erróneamente que está presente), negativos verdaderos (NV; el estímulo test no está presente en el conjunto de memorización y el sujeto experimental responde correctamente que no está presente) y falsos negativos (FN; el estímulo test está presente en el conjunto de memorización y el sujeto experimental responde que no está presente).

Tabla 2. Matriz de confusión

\begin{tabular}{|c|c|c|c|}
\cline { 3 - 4 } \multicolumn{2}{c|}{} & \multicolumn{2}{c|}{ Respuesta } \\
\cline { 3 - 4 } \multicolumn{2}{c|}{} & Correcta & Incorrecta \\
\hline \multirow{2}{*}{ Estímulo } & Presente & VP & FN \\
\cline { 2 - 4 } & Ausente & VN & FP \\
\hline
\end{tabular}

- Tiempo medio de respuesta en función del tipo de respuesta. Tiempo medio que tarda el sujeto experimental en presionar el botón en función de la categorización de la respuesta en la matriz de confusión. 


\subsubsection{Análisis de datos eye-tracking: efecto de recencia.}

Con el objetivo de analizar la interrelación entre la atención visual y la MT, se ha llevado a cabo un análisis específico de los datos de eye-tracking para evaluar si aquellos estímulos que se miran en las últimas posiciones de una secuencia tienen mayor probabilidad de ser recordados, es decir, si emerge un efecto de recencia. Para ello, sólo se han considerado los ensayos en que se muestran 6 colores y en los que el estímulo test se encuentra presente en el conjunto de memorización. Por tanto, en este estudio se trabaja sobre una base de 42 ensayos para cada sujeto.

Cabe destacar que el experimento se ha realizado bajo un paradigma experimental de búsqueda libre, de modo que los sujetos no reciben ninguna instrucción sobre cómo deben mirar el conjunto de memorización. Esto implica que aparezca una variedad de estrategias de visualización de los estímulos. La Fig. 2 muestra tres tipologías de exploración visual del conjunto de memorización diferentes: visualización periférica, visualización exhaustiva, visualización de mínima búsqueda.

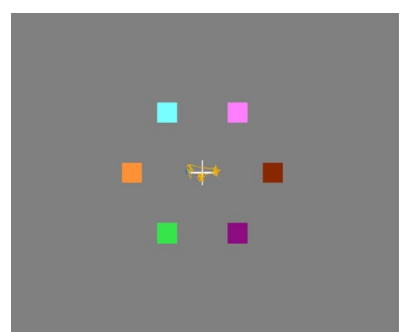

(a)

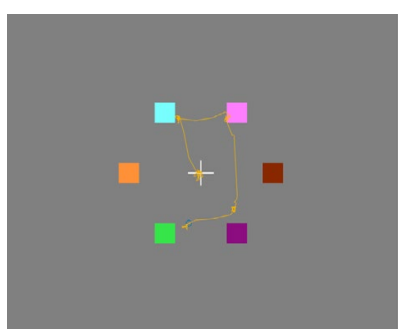

(b)

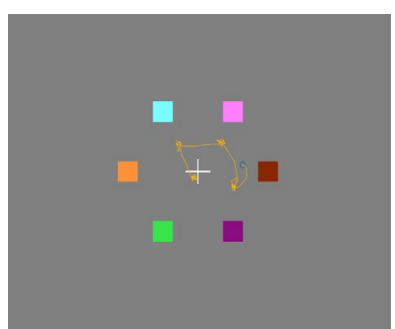

(c)

Fig. 2: Ilustración de tres estrategias de visualización diferentes durante la fase de memorización en la tarea DMS. La línea amarilla representa la trayectoria de exploración de la imagen que han seguido los sujetos en los tres ensayos considerados. (a) Visualización periférica: el sujeto mantiene la mirada en la zona central y explora los colores por medio de visión periférica, (b) Visualización exhaustiva: el sujeto observa de forma abierta y deliberada diversos estímulos durante la fase de memorización, y (c) Visualización de mínima búsqueda: el sujeto realiza una exploración abierta del conjunto de memorización pero no no fija la mirada en los estímulos.

En los análisis realizados en esta sección, sólo se han considerado las exploraciones de tipo exhaustivo. De los datos de eye-tracking se ha obtenido la secuencia de visualización de los diferentes estímulos. Un estímulo se considera procesado si el sujeto pasa un mínimo de $100 \mathrm{~ms}$ consecutivos en la zona de interés que, en nuestro estudio, se define como una región de 3 × 3 grados visuales centrados en cada estímulo. 


\section{Resultados}

\subsection{Rendimiento en la tarea DMS}

Tal y como se puede observar en Fig. 3(a), globalmente, los adultos jóvenes obtuvieron más respuestas correctas que los adultos de mediana edad $(t=3.108, p<0.005)$. Si se distingue entre el número de estímulos del conjunto de memorización, Fig. 3(b), se produjeron diferencias significativas entre las dos cohortes para 4 estímulos $(t=-3.031, p<0.005)$ y 6 estímulos $(t=-2.892, p=0.005)$ pero no para 2 estímulos. $(t=-0.675, p=0.502)$.

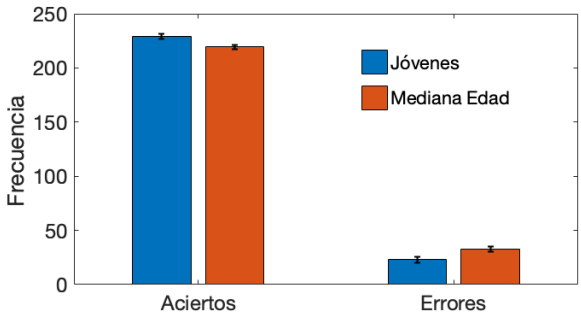

(a)

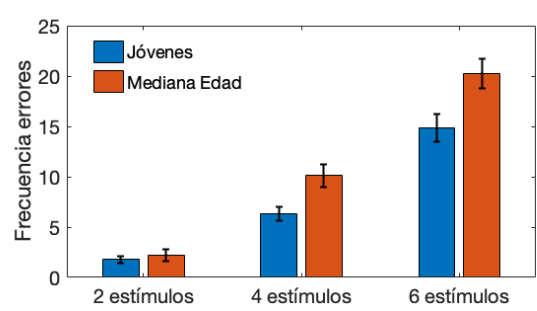

(b)

Fig. 3 (a) Frecuencia de errores y aciertos en la tarea DMS para las poblaciones $J$ y M, (b) frecuecnia de errores en la tarea para las poblaciones $J$ y M y variación en función del número de estímulos presentes en el conjunto de estimulación.

Tabla 3. Comparación del rendimiento de las poblaciones $\mathrm{J}$ y $\mathrm{M}$ en la tarea DMS.

\begin{tabular}{|ccc|}
\hline & $\boldsymbol{t}$-test & $\boldsymbol{p}$ \\
Aciertos & 3.108 & 0.003 \\
Errores & -3.108 & 0.003 \\
\hline
\end{tabular}

Tabla 4. Comparación del comportamiento de las poblaciones $\mathrm{J}$ y $\mathrm{M}$ en función de los errores cometidos para distintos tamaños del conjunto de memorización.

\begin{tabular}{|ccc|}
\hline \# Estímulos & $\boldsymbol{t}$-test & $\boldsymbol{p}$ \\
2 & -0.675 & 0.502 \\
4 & -3.031 & 0.004 \\
6 & -2.892 & 0.005 \\
\hline
\end{tabular}

Cuando se analizan por separado los tres bloques se observa que hay diferencias significativas en el rendimiento de ambas poblaciones en el primer bloque en que la población $\mathrm{J}$ muestra un rendimiento superior $(t=-3.983, p<0.001$, ver Tabla 5). Asimismo, si se atiende al rendimiento a lo largo de la tarea, se desprende que existen diferencias significativas entre el rendimiento en la tarea a lo largo de los bloques, tanto para la población de jóvenes adultos ( $\mathrm{J}$ ) (ANOVA medidas repetidas, $F=5.389, p=0.010$ ), como en la población de adultos de mediana edad (M) (ANOVA medidas repetidas $F=$ $22.914, p<0.001)$. Por tanto, se puede concluir que se produce un aprendizaje a lo largo de la tarea. 


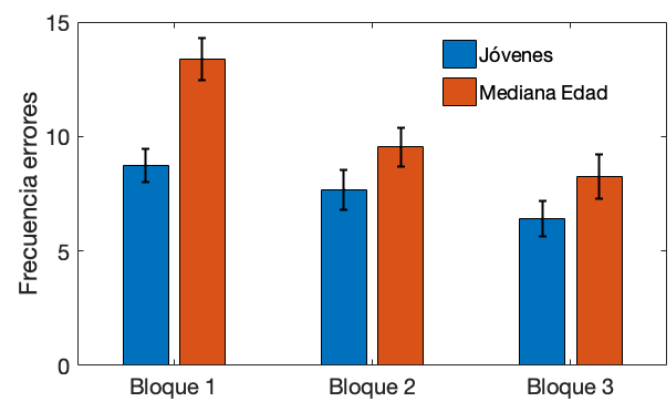

Fig. 4 Frecuencia de errores en la tarea DMS para las poblaciones $J$ y $M$ en función del bloque experimental. El bloque 1 se realiza en primer lugar, seguido de los bloques 2 y 3, consecutivamente.

Tabla 5. Comparación del rendimiento de las poblaciones $J$ y $\mathbf{M}$ en función del bloque experimental.

\begin{tabular}{|ccl|}
\hline & $\boldsymbol{t}$-test & \multicolumn{1}{c|}{$\boldsymbol{p}$} \\
1 & -3.983 & $<0.001$ \\
2 & -1.488 & 0.143 \\
3 & -1.473 & 0.147 \\
\hline
\end{tabular}

Otro modo de analizar y de comparar el rendimiento de las dos poblaciones es atendiendo a la tipología de los aciertos y de los errores (ver Tablas 6 y 7). Aunque el número de aciertos de la población joven aumenta respecto al de la población de mediana edad, esta diferencia no alcanza valores estadísticamente significativos ni en el caso de PV ni en el de FN. Sin embargo, sí que se aprecian diferencias significativas en el caso de los $\mathrm{FP}(t=-3.360, p=$ $0.001)$ y NV ( $t=-3.360, p=0.001)$ lo cual puede interpretarse como un empobrecimiento en la capacidad de codificar la información que llega a los sentidos con la edad.

Tabla 6. Matriz confusión promedio obtenida a partir de datos comportamentales de la población joven.

\begin{tabular}{|l|c|c|c|}
\cline { 3 - 4 } \multicolumn{2}{c|}{} & \multicolumn{2}{c|}{ Respuesta } \\
\cline { 3 - 4 } \multicolumn{2}{c|}{} & Correcta & Incorrecta \\
\hline \multirow{2}{*}{ Estímulo } & Presente & 117.17 & 7.97 \\
\cline { 2 - 4 } & Ausente & 118.03 & 14.83 \\
\hline
\end{tabular}

Tabla 7. Matriz confusión confusión promedio obtenida a partir de datos comportamentales de la población mediana edad.

\begin{tabular}{|c|c|c|c|}
\cline { 3 - 4 } \multicolumn{2}{c|}{} & \multicolumn{2}{c|}{ Respuesta } \\
\cline { 3 - 4 } \multicolumn{2}{c|}{} & Correcta & Incorrecta \\
\hline \multirow{2}{*}{ Estímulo } & Presente & 109.67 & 19.42 \\
\cline { 2 - 4 } & Ausente & 111.17 & 16.33 \\
\hline
\end{tabular}

Asimismo, si nos fijamos en los tiempos que se tarda en emitir una respuesta, no se observan diferencias significativas entre ambas poblaciones para ninguno de los tipos de situaciones identificadas en la matriz de confusión (i.e. $p>0.05$ en todos los casos). Sin embargo, llama la atención que las respuestas incorrectas, y especialmente, los FN tienen 
asociados tiempos de reacción mayores, sugiriendo de este modo que la información visual ha sido codificada en algún nivel (ver Tablas 8 y 9).

Tabla 8. Matriz confusión promedio obtenida a partir de datos comportamentales de la población joven.

\begin{tabular}{|c|c|c|c|}
\cline { 3 - 4 } \multicolumn{2}{c|}{} & \multicolumn{2}{c|}{ Respuesta } \\
\cline { 3 - 4 } \multicolumn{2}{c|}{} & Correcta & Incorrecta \\
\hline \multirow{2}{*}{ Estímulo } & Presente & $0.46 \mathrm{~s}$ & $1.18 \mathrm{~s}$ \\
\cline { 2 - 4 } & Ausente & $0.51 \mathrm{~s}$ & $0.89 \mathrm{~s}$ \\
\hline
\end{tabular}

Tabla 9. Matriz confusión confusión promedio obtenida a partir de datos comportamentales de la población mediana edad.

\begin{tabular}{|c|c|c|c|}
\cline { 3 - 4 } \multicolumn{2}{c|}{} & \multicolumn{2}{c|}{ Respuesta } \\
\cline { 3 - 4 } \multicolumn{2}{c|}{ Estímulo } & Correcta & Incorrecta \\
\cline { 2 - 4 } & Presente & $0.50 \mathrm{~s}$ & $1.06 \mathrm{~s}$ \\
\hline
\end{tabular}

\subsection{Efecto recencia}

En relación a los efectos seriales, el estudio se centró en aquellos ensayos en que se mostraron 6 estímulos. De éstos, en el 26\% de los 84 ensayos/persona tuvieron lugar búsquedas exhaustivas (las secuencias de fijaciones están totalmente contenidas en ROIs de $3 \times 3$ grados visuales centrados en los estímulos). Con el objetivo de analizar efectos seriales se han considerado aquellas secuencias que tienen una longitud $N>2$. En nuestro estudio, $N_{\max }=4$. Por tanto, se han analizado (de forma independiente para cada tamaño) las secuencias que contienen 3 y 4 fijaciones. Finalmente, se han agregado los datos de las secuencias de longitud 3 y 4 , alineando las secuencias respecto al último estímulo observado. Esto permite observar si existe un efecto serial en las posiciones $F, F-1$ y $F-$ 2, siendo $F$ la última posición de la secuencia, $F$-1 la penúltima y $F-2$ la antepenúltima.

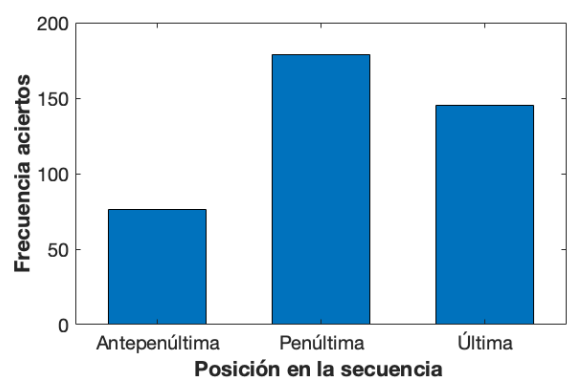

Fig. 5: Efectos seriales en el rendimiento de la tarea. Se muestra la frecuencia con que los sujetos experimentales han dado una respuesta correcta en función de la posición en la secuencia en que han observado el estímulo test.

Tal y como se puede observar en la Fig. 5, los 2 últimos estímulos que recibieron atención abierta durante la búsqueda fueron reconocidos significativamente mejor que los estímulos visitados con anterioridad durante la fase de codificación (desde la posición final, $1^{\mathrm{o}}$ vs $2^{\mathrm{o}}$ $\chi^{2}=2.382 p=0.140 ; 2^{\circ}$ vs $3^{\circ} \chi^{2}=28.248, p<0.001$ ) sugieriendo de este modo la aparición de 
un efecto de recencia y poniendo de relieve la importancia de la interacción entre el despliegue de atención visual y la MT.

\section{Conclusiones}

En este trabajo se ha investigado la memoria de trabajo en el marco de una tarea DMS y su deterioro con la edad. Para ello, se han reclutado dos grupos de sujetos de diferente edad (jóvenes adultos, $\mathrm{J}$, y adultos de mediana edad, M) y se ha diseñado un protocolo experimental que permite combinar la tarea DMS con medidas de eye-tracking. Los resultados obtenidos están de acuerdo con las previsiones de estudios computacionales anteriores llevados a cabo en nuestro grupo (Dempere-Marco et al, 2012; Rolls et al., 2013, Balagué y Dempere-Marco, 2016). En particular, las predicciones de los modelos computacionales indican que la memoria de trabajo yace en un continuo entre las predicciones de los modelos slot (Zhang y Luck, 2008) y los modelos de recursos compartidos (Bays y Husain, 2008). En este contexto, la atención visual desempeña un papel crucial tal y como se investigó en términos de la relevancia visual y su rol en los procesos atencionales de carácter bottom-up en (Piazza y Melcher, 2011) y (DempereMarco et al., 2012).

En primer lugar, nuestros resultados aportan evidencia experimental sobre la disminución de la capacidad de MT con la edad y con el tamaño del conjunto de memorización. Además, en este estudio la introducción de técnicas de eye-tracking en el protocolo experimental permite analizar cómo se despliega la atención visual abierta durante el período de codificación de los estímulos. Los resultados obtenidos respaldan la hipótesis de que la atención visual modula la codificación de los elementos en MT. En particular, el efecto de recencia surge como consecuencia de la asignación de atención visual a los diferentes elementos durante la fase de estimulación de la tarea DMS, lo que ilustra el papel tan importante que tiene la interacción entre la atención visual y MT.

\section{Agradecimientos}

Las autoras agradecen a Laura Portell su colaboración en el proyecto de investigación en el marco del cual contribuyó en el diseño del protocolo experimental de la tarea DMS. Esta investigación ha sido posible gracias a la financión recibida por parte Ministerio de Ciencia e Innovación a través del proyecto TIN2013-40630-R.

\section{Referencias}

BALAGUE, M., DEMPERE-MARCO, L. (2016) Multi-item Working Memory Capacity: What Is the Role of the Stimulation Protocol? In Lecture Notes in Computer Science Vol. 9886: 264-271. 
Evaluación de la interacción entre atención visual y memoria de trabajo: un estudio basado en técnicas de eye-tracking

BAYS, P., HUSAIN, M., (2008) "Dynamic shifts of limited working memory resources in human vision" en Science Vol. 321, pp. 851-854.

CASALS-COLL, M. (2013) "Estudios normativos españoles en población adulta joven (proyecto NEURONORMA jóvenes): normas para los test de fluencia verbal” en Neurología, Vol. 28 (1), pp. 33-40.

COALSON D. L. RAIFORD S. E. WECHSLER, D. (2008) "WAIS-IV: Wechsler adult intelligence scale", San Antonio TX Pearson.

COWAN, N. (2000) "The magical number 4 in short-term memory: A reconsideration of mental storage capacity" in Behavioural Brain Science, Vol. 24, pp. 87-116.

COWAN, N. (2017) "The many faces of working memory and short-term storage" in Psychonomic Bulletin Review, Vol. 24, pp. 1158-1170.

DEMPERE-MARCO, L., HU, X-P., YANG, G.Z., (2011) "A novel framework for the analysis of eye movements during visual search for knowledge gathering" en Cognitive Computation, Vol. (3) 1, pp. 206-222

DEMPERE-MARCO, L., MELCHER, D. P., DECO, G. (2012) "Effective visual working memory capacity: an emergent effect from the neural dynamics in an attractor network" en PLoS One, Vol. 7 (8): e42719. https://doi.org/10.1371/journal.pone.0042719 [Consulta: 15 de mayo de 2019]

GOLDEN, CJ. (1978) "Stroop Color and Word Test: a manual for clinical and experi- mental uses", Wood Dale: Stoeling Company.

MELCHER, D., PIAZZA, M. (2011) "The role of attentional priority and saliency in determining capacity limits in enumeration and visual working memory" en PLoS One Vol. 6: 1-11 [Consulta: 15 de mayo de 2019]

PALLARES, V., HERNANDEZ, M., DEMPERE-MARCO, L. (2017) "Eye-Tracking Data in Visual Search Tasks: A Hallmark of Cognitive Function" en Converging Clinical and Engineering Research on Neurorehabilitation II, 873-877.

PALLARES, V., RAMI, L., DEMPERE-MARCO, L. (2016), “A Novel Method for Disentangling Strategies from Visual Search“" en BIOSIGNALS, 271-276

PEÑA-CASANOVA, J., QUIÑONES-UBEDA, S., QUINTANA-APARICIO, M., AGUILAR, M., BADENES, D., MOLINUEVO, JL., TORNER, L., ROBLES, A., BARQUERO, MS., VILLANUEVA, C., ANTUNEZ, C., MARTINEZ-PARRA, C., FRANK-GARCIA, A., SANZ, A., FERNANDEZ, M., ALFONSO, V., SOL, JM., BLESA, R. (2009a) "Spanish Multicenter Normative Studies (NEURONORMA Project): norms for verbal span, visuospatial span, letter and number sequencing, trail making test, and symbol digit modalities test", en Archives of Clinical Neuropsychology, Vol. 24 (4), pp. 321-341.

PEÑA-CASANOVA, J., QUIÑONES-UBEDA, S., QUINTANA-APARICIO, M., AGUILAR, M., BADENES, D., MOLINUEVO, JL., TORNER, L., ROBLES, A., BARQUERO, MS., VILLANUEVA, C., ANTUNEZ, C., MARTINEZ-PARRA, C., FRANK-GARCIA, A., SANZ, A., FERNANDEZ, M., ALFONSO, V., SOL, JM., BLESA, R. (2009b) "Spanish Multicenter Normative Studies (NEURONORMA Project): norms for the Stroop color-word interference test and the Tower 
of London-Drexel", en Archives of Clinical Neuropsychology, Vol. 24 (4), pp. 413-429.

PEÑA-CASANOVA， J., QUIÑONES-ÚBEDA， S., GRAMUNT-FOMBUENA， N., QUINTANA-APARICIO, M., AGUILAR, M., BADENES, D., BARQUERO, M. S. (2009C). "Spanish Multicenter Normative Studies (NEURONORMA Project): norms for verbal fluency tests" en Archives of Clinical Neuropsychology, Vol. 24 (4), pp. 395-411.

ROGNONI, T. (2004) "Estudios normativos españooles en población adulta joven (proyecto NEURONORMA jóvenes) normas para las pruebas Stroop Color-Word Interference Test y Tower of London-Drexel University” en Psychological Sciences, Vol. 15:106-111.

ROLLS, E.T., DEMPERE-MARCO, L., DECO, G. (2013) "Holding multiple items in short term memory: a neural mechanism" en PLoS One, Vol. 8 (4): e61078. https://doi.org/10.1371/journal.pone.0061078 [Consulta: 15 de mayo de 2019]

SPREEN, O., STRAUSS, E., SHERMAN, EMS. (2006) “A compendium of neuropsychological test. Administration, norms, and commentary” New York: Oxford University Press.

TAMAYO, F. (2012) "Estudios normativos españoles en población adulta joven (Proyecto NEURONORMA jóvenes): normas para las pruebas span verbal, span vi- suoespacial, Letter-Number Sequencing, Trail Making Test y Symbol Digit Modalities Test” en Neuroloía, Vol. 27 (6), pp. 319 329.

YANG, GZ., DEMPERE-MARCO, L., HU, XP., ROWE, A. (2002) "Visual search: psychophysical models and practical applications" en Image and Vision Computing, Vol. 20 (4), pp. 291-305

ZHANG, W., LUCK, S. (2008) "Discrete fixed-resolution representations in visual working memory" en Nature, Vol. 453, pp. 233-235. 